\title{
Spectroscopy of the Potential Profile in a Ballistic Quantum Constriction
}

\author{
I. E. Aronov', M. Jonson, and A. M. Zagoskin \\ Department of Applied Physics, Chalmers University of Technology and University of Göteborg, S-412 96 Göteborg, Sweden
}

\begin{abstract}
We present a theory for the nonlinear current-voltage characteristics of a ballistic quantum constriction. Nonlinear features first develop because of above-barrier reflection from the potential profile, created by impurities in the vicinity of the constriction. The nonlinearity appears on a small voltage scale and makes it possible to determine distances between impurities as well as the magnitude of the impurity potentials.
\end{abstract}

72.10.Bg, 05.60.tw, 73.40.Kp

In recent experiments on microconstrictions, electrostatically created by a split-gate technique in the $2 \mathrm{D}$ electron gas of a GaAs-AlGaAs heterostructure, several groups [1.2] have reported small-scale nonlinear features of the system conductance when measured as function of the driving voltage, $V$. These anomalies, which occurred on the first fundamental $2 e^{2} / h$ - conductance plateau, still lack a satisfactory explanation. In this Letter we show that the small-scale nonlinearity in the conductance is decisively determined by the shape of the effective potential barrier inside the quantum ballistic constriction (QBC). The latter is defined by the geometry of the microconstriction and by impurities in its vicinity. The effective potential profile leads to an 'above-barrier' reflection effect [3], which gives rise to the nonlinearity in the QBC under consideration. In short, the characteristic scale of the nonlinear features is determined by the characteristic inter-impurity distances.

Our starting point is the well known adiabatic model [4] of a QBC shown in Fig. 15. Numerical calculations [5,6] confirm that the effect of charged impurities in the vicinity of the microconstriction is to smoothly modulate the electrostatic potential inside the constriction, $V_{i m p}(x, y)$. This modulation can be thought of as a simultaneous modulation of the microconstriction geometry and a smooth variation of the effective onedimensional potential for longitudinal transport along the QBC axis. Such a picture is valid as long as the condition $\left|\partial V_{i m p} / \partial y\right| d(0) \ll \Delta E_{\perp}$ is satisfied. Here $\Delta E_{\perp} \simeq \pi^{2} \hbar^{2} / 2 m^{*} d(0)^{2}$ is a characteristic intermode level spacing in the adiabatic quantum constriction.

Assuming the constriction to be long and smooth enough, the criterion $d^{\prime}(x) \simeq d(0) / l \ll 1$ is fullfilled ( $l$ is the length of the contact), and we are in the adiabatic transport regime. Under these conditions - to zero order in corrections to adiabatic transport - the Schrödinger equation for the longitudinal part of the wave function in the $n$-th transverse mode is

$$
-\frac{\hbar^{2}}{2 m^{*}} \psi_{n}^{\prime \prime}(x)+W_{n}(x) \psi_{n}(x)=E \psi_{n}(x) .
$$

Here $W_{n}(x)=V_{i m p}(x, 0)+E_{n \perp}(x)$ corresponds to an effective gate-generated potential profile modulated by the impurity potential both directly $\left(V_{i m p}(x, 0)\right)$ and through the modulation of the constriction width, which depends on the transverse component of the impurity potential.

We consider an electron in the $n$-th propagating mode, and assume that the electron energy is everywhere much higher than the effective potential, $E \gg W_{n}(x)$. In this case above-barrier scattering will lead to a reflection coefficient of the form [3]

$$
R_{n}(E)=\left|m^{*} /\left(\hbar^{2} k(E)\right) \int_{-\infty}^{\infty} W_{n}(x) e^{2 i k(E) x} d x\right|^{2},
$$

where $k(E)=\sqrt{2 m^{*} E} / \hbar$.

The current through the contact is given by [7]

$$
\begin{aligned}
& I(V)=(2 e / h) \int d E\left[n_{F}\left(E-\mu_{1}\right)\right. \\
& \left.-n_{F}\left(E-\mu_{2}\right)\right] \sum_{n=1}^{N}\left(1-R_{n}(E)\right) .
\end{aligned}
$$

Here $N$ is a number of propagating modes, $\mu_{1}=E_{F}-$ $\beta e V$ and $\mu_{2}=E_{F}+(1-\beta) e V$ are the chemical potentials of the left- and right electron reservoirs, and $0 \leq \beta \leq 1$ is factor that describes the asymmetry of the voltage drop along the constriction [8].

The most spectacular situation occurs at zero temperature, when a simple expression for the differential conductance of the system follows from Eq. (3). One finds that

$$
G(V)=N\left(2 e^{2} / h\right)-\delta G_{N}(V)
$$

where

$$
\begin{aligned}
\delta G_{N}(V) & =\left(2 e^{2} / h\right) \sum_{n=1}^{N}\left[\beta R_{n}\left(E_{F}-\beta e V\right)\right. \\
& \left.+(1-\beta) R_{n}\left(E_{F}+(1-\beta) e V\right)\right] .
\end{aligned}
$$

Clearly, the reflection coefficient (2) is determined by two factors; first by the contact geometry, and second by the impurity potential. The geometric contribution to $W_{n}(x)$, i.e. $E_{n \perp}(x)$, is a sum of a smooth function and a small-scale oscillatory term. The former is due to the gate potential, while the latter is induced by the transverse part of the impurity potential. It is well known 
that the smooth part of $E_{n \perp}(x)$ contributes an exponentially small term to $R_{n}$ 肺. Nevertheless, as the number of propagating modes increases, the total amplitude due to this contribution grows as $N^{5}$ (as $\left.E_{n \perp} \propto n^{2}\right)$. Therefore, if only the number of open modes becomes large enough, the relative amplitude of the oscillatory component of the reflection coefficient is weakened. The best conditions for doing 'potential profile spectroscopy' are then at hand when the number of propagating modes is as small as possible.

From now on we take $N=1$. We can thus neglect the contribution to $R_{n}$ from the adiabatic part of the transverse energy that enter $W_{n}(x)$. Evidently, the other contributions to this function, coming from the irregularities of the constriction geometry and from the potential profile inside the constriction, can not be separated. This corresponds to the physical situation, since both these contributions have the same origin: the random potential of the adjacent impurities. We denote this part of the potential $W_{n}(x)$ - which is independent of mode index $n$ - by $\tilde{V}_{i m p}(x)$.

Note that by considering only a single propagating mode we automatically exclude opening/closing of conducting modes [1,9,10] as well as coherent mode mixing 11] as possible mechanisms for the nonlinear features discussed.

The effective potential $\tilde{V}_{i m p}(x)$ is written in the form

$$
\tilde{V}_{i m p}(x)=\sum_{\alpha} U_{\alpha}\left(\left|x-x_{\alpha}\right|\right)
$$

where $U_{\alpha}$ denotes the contribution to the self-consistent effective potential from the $\alpha$-th impurity, situated at the point $x_{\alpha}$. The reflection coefficient then acquires the simple form

$$
\begin{aligned}
R(E) & =\left(m^{*} / \hbar^{2} k(E)\right)^{2}\left|\sum_{\alpha} e^{2 i k(E) x_{\alpha}} u_{\alpha}(E)\right|^{2}, \\
u_{\alpha}(E) & =\int_{-\infty}^{\infty} d x e^{2 i k(E) x} U_{\alpha}(|x|)
\end{aligned}
$$

where $u_{\alpha}(E)$ is the Fourier transform of the effective impurity potential.

It is evident from (7) that the reflection coeffitient is sensitive to the interimpurity distances. It follows from the uncertainty condition for the direct and inverse Fourier transform that the accuracy $\Delta x$ with which these distances can be determined from (7) is limited by $\Delta x \Delta k \geq 1 / 4 \pi$. In our case this reduces to

$$
\Delta x>\simeq \lambda_{F} E_{F} /\left(4 \pi^{2}|e V|\right)
$$

The small numerical factor $1 / 4 \pi^{2}$ allows for relatively small driving voltages to define the interimpurity distances with accuracy of order $\lambda_{F}$.
It is easy to estimate from Eq.(7) the order of magnitude of the relative conductance variation due to abovebarrier scattering at zero temperature:

$$
\frac{\delta G}{G} \simeq\left(\pi \frac{l_{i m p}}{\lambda_{F}} \frac{U_{i m p}}{E_{F}}\right)^{2}
$$

Because of the smoothness of the effective impurity potential the quantity $l_{i m p} U_{i m p}$ is the maximal value of its Fourier transform, $l_{i m p}$ being its effective length, and $U_{i m p}$ its magnitude. The characteristic scales for these quantitites are given by different authors in a rather wide interval. Following [6], we use $U_{i m p} / E_{F} \simeq 0.3 ; l_{i m p} / \lambda_{F}$ can be estimated to be in the range 0.1 to 0.01 . That is, the magnitude of the nonlinear contribution to the conductance at zero temperature is $1-10^{-2} \%$.

At finite temperatures the conductance oscillations will be washed out. Thus in order to observe the effect described it is necessary that $k_{B} T \ll e \Delta V$, where $\Delta V$ is the period of oscillation with bias voltage. A realistic estimate is that the effect can be observed if $T \leq 100 \mathrm{mK}$.

Numerical calculations of the conductance vs. bias voltage are presented in Figs. 2f 4. We use Coulomb impurity potentials,

$$
U_{\alpha}\left(\left|x-x_{\alpha}\right|\right)=Z_{\alpha} e^{2} / \kappa \sqrt{\left(x-x_{\alpha}\right)^{2}+h_{\alpha}^{2}} .
$$

The second term under the square root determines the lateral and vertical displacement of the $\alpha$-th impurity from the axis of the constriction. The dielectric constant for GaAs is $\kappa \approx 13$. An asymmetric voltage drop $(\beta \neq$ 0.5 ) leads to an asymmetric response $\delta G(V)$. Evidently the larger the inter-impurity distance, the more distinct is the picture of oscillations, and the larger is the accuracy with which we can determine the potential profile (see Eq.(8)).

The average value of the nonlinear term in the conductance is described by the averaged value of the function $R(E) \cdot E$ (see Eq. (2)). Making use of the Parseval inequality, we obtain the result

$$
\langle R(E) E\rangle=\frac{1}{2 \pi} \int_{-\infty}^{\infty} d k R(E) E=\frac{m^{*}}{4 \hbar^{2}} \int_{-\infty}^{\infty} d x\left|\sum_{\alpha} U_{\alpha}(x)\right|^{2} .
$$

In this way we find the average magnitude of the impurity potential along the axis of the constriction.

Since the Fourier transform of $R(k(E)) E$ directly gives the set of interimpurity distances, $\Delta x_{\alpha \alpha^{\prime}}$, we can apply a filtration procedure on the corresponding frequencies, that is find

$$
\langle R E\rangle_{\Delta x_{\alpha \alpha^{\prime}}}=\frac{1}{2 \pi} \int_{-\infty}^{\infty} d k R(k) E(k) \exp \left(-2 i k \Delta x_{\alpha \alpha^{\prime}}\right) .
$$


Hence we can analyze the lineshape of every peak in the trace of $\delta G(V)$, initiated by each distinguishable impurity. In the general case, such an analysis allows us to determine the quantities $\left|h_{\alpha}-h_{\alpha^{\prime}}\right| /\left|h_{\alpha}+h_{\alpha^{\prime}}\right|$, thus providing information about the distances between the impurities and the constriction plane.

Using the model potentials 10 we find for the line shape an expression $\langle R E\rangle_{\Delta x_{\alpha \alpha^{\prime}}}=\left(m^{*} / \hbar^{2}\right)\left(e^{4} Z_{\alpha} Z_{\alpha^{\prime}} / \kappa^{2}\left|h_{\alpha}+h_{\alpha^{\prime}}\right|\right)$. $\mathbf{K}\left(\left|h_{\alpha}-h_{\alpha^{\prime}}\right| /\left|h_{\alpha}+h_{\alpha^{\prime}}\right|\right)$. The function $\mathbf{K}(\xi)$ is the complete elliptic integral of the first kind.

The nonlinear contribution $\delta G(V)$ to the conductance allows us to determine the spectral characteristics of the potential profile created by impurities in the constriction. For real impurity potentials [5, 6] $U_{\alpha}(k)$ are smooth functions of the variable $k$ and therefore of the driving voltage $V$. Because of this fact we can apply the Fourier transformation technique to Eq.(5) and from the positions of the spectral peaks determine the distances $\Delta x_{\alpha \alpha^{\prime}}=\left|x_{\alpha}-x_{\alpha^{\prime}}\right|$ between the impurities. Evidently, these distances can be measured only if they are larger than $\lambda_{F}$. If on the contrary $\Delta x_{\alpha \alpha^{\prime}} \leq \lambda_{F}$ we can regard the corresponding impurity cluster as a single scatterer. Anyhow, it follows from (5) that they can be resolved only at large enough voltages $V$, i.e., in the regime where the nonlinear effects are developed. A Fourier analysis of $G(V)$ as well permits us to find the quantities [12] $\sum_{\alpha}\left|U_{\alpha}\right|^{2}$ and $\sum_{\alpha \neq \alpha^{\prime}} U_{\alpha} U_{\alpha^{\prime}}^{*} \cos \left(\operatorname{Arg}\left(U_{\alpha} U_{\alpha^{\prime}}^{*}\right)\right)$, which characterize the magnitudes of the impurity potentials.

In Figs.24 different types of conductance-voltage curves caused by above-barrier reflection in the constriction are shown. We plot there the nonlinear contribution $\delta G$ to the differential conductance(in arbitrary units) vs. the reduced driving voltage $v=V / E_{F}$. The scale of $v \in[-0.5 ; 0.5]$ is consistent with measured values of $E_{F} \sim 10 \mathrm{meV}$ and distance between the first and second subbands in a QBC, $\Delta E_{\perp} \simeq 5 \mathrm{meV}$ [1]. The potential drop is assumed to be completely asymmetric $(\beta=1)$.

In Fig. 2 the next simplest case is shown, with a single impurity. Evidently the nonlinear correction is a monotonic function of voltage. Nonmonotonitic behavior appears when two impurities are present (Fig.3p. Fig.4 demonstrates the case of three impurities. It is easy to see that the structure of the curves becomes richer as the number of impurities and/or average distance between them grows.

In conclusion, we have demonstrated that abovebarrier scattering of electrons by the potential profile in quantum ballistic constrictions leads to small-scale nonlinearities in the current-voltage characteristics. The effect is best observed when only one conducting channel is open, and at temperatures less than $\simeq 100 \mathrm{mK}$. It can be used to determine the spatial arrangement of the impurities in the constriction.

This work was supported by the Swedish Academy of
Sciences (KVA), the Natural Science Research Council (NFR), and by NUTEK. We are grateful to P. E. Lindelof, L. Kuzmin, M. Persson, and R. I. Shekhter for productive discussions. One of us (IA) acknowledges the hospitality of the Department of Applied Physics at $\mathrm{CTH} / \mathrm{GU}$.

* Permanent address: Institute of Radiophysics and Electronics, Ukrainian Academy of Sciences, Acad. Proscura St. 12, 310085 Kharkov, Ukraine.

[1] N. K. Patel, J. T. Nicholls, L. Martin-Moreno, M. Pepper, J. E. F. Frost, D. A. Ritchie, and G. A. C. Jones. Phys.Rev. B 44 (1991) 13549.

[2] R. Taboryski, A. K. Geim, and P. E. Lindelof. Superlattices and Microstructures 12, 137 (1992). R. Taboryski, A. K. Geim, M. Persson, and P. E. Lindelof, Phys. Rev. $\mathrm{B}$ (in press).

[3] L. D. Landau, E. M. Lifshits. Quantum mechanics (Pergamon, Oxford, 1977).

[4] L. I. Glazman, G. B. Lesovik, D. E. Khmel'nitskii, and R. I. Shekhter. Pis'ma Zh. Eksp. Teor. Fiz. 48, 218 (1988) [JETP Lett. 48, 238 (1988)].

[5] M.J. Laughton, J. R. Barker, J. A. Nixon, and J. H. Davies. Phys.Rev. B 44, 1150 (1991); J.A. Nixon and J.H. Davies. Phys.Rev.B 41, 7929 (1990).

[6] S.N. Rashkeev, A.M. Zagoskin, R.I. Shekhter, and G. Wendin, to be published.

[7] Y.Imry, in Directions in Condensed Matter Physics, ed. by G.Grinstein and G.Mazenko (World Scientific Publ., Singapore, 1986), p.101.

[8] L. Martin-Moreno, J. T. Nicholls, N. K. Patel, and M. Pepper. J. Phys.: Condens. Matter, 4, 1323 (1992).

[9] A. M. Zagoskin. Pis'ma Zh.Eksp.Teor.Fiz. 52 (1990) 1043 [JETP Lett. 52 (1991) 435].

[10] L. P. Kouwenhoven, B. J. van Wees, C. J . P . M. Harmans, J. G. Williamson, H. van Houten, C. W. J. Beenakker, C. T. Foxon, and J. J. Harris. Phys.Rev. B 39 (1989) 8040.

[11] A.M.Zagoskin and R.I.Shekhter, Phys.Rev.B (in press).

[12] R.H.T.Bates and M.J.McDonnell. Image Restoration and Reconstruction, Clarendon Press, Oxford, 1986.

FIG. 1. Quantum ballistic constriction. (a) Clean adiabatic channel; $d(x)$ is a smooth function of longitudinal coordinate. (b) Effective potential profile inside the channel in presence of impurities.

FIG. 2. Nonlinear contribution to the conductance of the constriction with a single impurity. 
FIG. 3. Nonlinear contribution to the conductance of the constriction with two impurities. The oscillations arise due to Fabry - Perot type interference of electron wave between them (the distance between the impurities is $11 \lambda_{F}$ ).

FIG. 4. Nonlinear contribution to the conductance calculated for 3 impurities in the constriction. The average distance between the impurities equals to $23 \lambda_{F}$. 